\title{
Temperature dependence of the pre-edge structure in the Ti K-edge x-ray absorption spectrum of rutile
}

\author{
O. Durmeyer, E. Beaurepaire, J.-P. Kappler \\ IPCMS, UMR 7504 CNRS-ULP, 23 rue de Loess, B.P. 43 Strasbourg Cedex 2, France
}

Ch. Brouder

Institut de Minéralogie et de Physique des Milieux Condensés, CNRS UMR 7590,

Universités Paris 6 et 7, IPGP, 140 rue de Lourmel, 75015 Paris, France

F. Baudelet

Synchrotron SOLEIL, L'Orme des Merisiers, Saint-Aubin - BP 48, F-91192

Gif-sur-Yvette cedex, France.

E-mail: christian.brouder@impmc.jussieu.fr

\begin{abstract}
The temperature dependence of the pre-edge features in x-ray absorption spectroscopy is reviewed. Then, the temperature dependence of the pre-edge structure at the $\mathrm{K}$-edge of titanium in rutile $\mathrm{TiO}_{2}$ is measured at low and room temperature. The first two peaks grow with temperature. The fact that these two peaks also correspond to electric quadrupole transitions is explained by a recently proposed theory.
\end{abstract}

31 October 2018

PACS numbers: 78.70.Dm, 63.70.+h 


\section{Introduction}

Pre-edge peaks often arise at the K-edge of transition metal elements. This pre-edge structure is sensitive to the metal valence, to the symmetry of its surroundings and to the atomic species of the neighbours (see [1] for a recent review). As a consequence, the measurement and analysis of the pre-edge peaks are widely used in earth sciences [2], biology [3], chemistry [4] and physics [5, 6].

Because of their practical importance, pre-edge features have to be well understood and they were the object of detailed theoretical works using various approaches: multiplets [7, 8], Bethe-Salpeter equation [9], multiple-scattering [10], pseudopotential theory [5, 11]. Vedrinskii and his group were particularly active to extract information from the pre-edge structure [12, 13, 14].

In section 2, we make a short review of the literature to show that the temperature dependence of pre-edge peaks is not a rare property of x-ray absorption spectra. However, this dependence is usually attributed to static off-center displacements or to phase transitions. Therefore, our preliminary investigation [15] showing a temperature dependence of the pre-edge peaks at the titanium $\mathrm{K}$-edge in $\mathrm{TiO}_{2}$ (rutile) came out as a surprise because the pre-edge variation was observed in a temperature range where no phase transition occurs and where many high-precision structural studies [16, 17, 18, 19, 20] indicate that no off-center atomic displacement takes place. Soft modes have indeed be reported [21] but the calculated phonon spectrum shows excellent agreement with experiment and no imaginary mode is present [22, 23, 24, 25] when the proper functionals are used [26].

Thus, we carried out detailed experiments to confirm and analyze this temperature dependence. The results of these experiments are presented in section 3. Section 4 describes why such a temperature dependence is a priori surprising and sketches a theoretical interpration that enables us to understand why the temperature dependence is restricted to the first two peaks and why no energy shift is observed. A conclusion summarizes our results and provides possible extensions of this work.

\section{A short review}

In this section, we present a short and non-exhaustive review of the temperature dependence of pre-edge peaks.

As far as we know, such a temperature dependence was first observed by Durmeyer and coll. [15] at the K-edge of titanium in $\mathrm{TiO}_{2}$ (rutile), $\mathrm{Li}_{4 / 3} \mathrm{Ti}_{5 / 3} \mathrm{O}_{4}$ and $\mathrm{LiTi}_{2} \mathrm{O}_{4}$. It was subsequently measured at the titanium K-edge of a several perovskite crystals: $\mathrm{PbTiO}_{3}$ [27, 28, 12, 29, 30], $\mathrm{SrTiO}_{3}$ [31, 30], $\mathrm{BaTiO}_{3}$ [30, $\mathrm{CaTiO}_{3}$ [30].

A similar temperature dependence was observed at other edges in perovskite crystals: at the niobium K-edge of $\mathrm{KNbO}_{3}$ [32, 33], $\mathrm{NaNbO}_{3}$ [34, $\mathrm{PbIn}_{1 / 2} \mathrm{Nb}_{1 / 2} \mathrm{O}_{3}$ [35], at the zirconium K-edge of $\mathrm{PbZrO}_{3}$ [14], $\mathrm{PbZr}_{0.515} \mathrm{Ti}_{0.485} \mathrm{O}_{3}$ [14], $\mathrm{BaZrO}_{3}$ [14], at the K-edge of $\mathrm{Mn}$ in $\mathrm{La}_{1-x} \mathrm{Ca}_{x} \mathrm{MnO}_{3}$ [36, 37] and at the K-edge of $\mathrm{Fe}$ in in $\mathrm{La}_{0.8} \mathrm{Sr}_{0.2} \mathrm{FeO}_{3}$ 
and $\mathrm{La}_{0.7} \mathrm{Sr}_{0.2} \mathrm{FeO}_{3}[38$. In most cases, the temperature effect was intepreted in terms of a phase transition or of a static off-center atomic displacement due to the presence of very soft modes in the crystal.

However, the effect is not restricted to the perovskite structure. Apart from the results of Durmeyer and coll. [15], it was observed at the K-edge of titanium in $\mathrm{TiO}_{2}$ and $\mathrm{Mg}_{2} \mathrm{TiO}_{4}$ [30], at the L-edges of La in Sr-doped $\mathrm{La}_{2} \mathrm{CuO}_{4}$ [39] and at the K-edge of $\mathrm{V}$ in $\mathrm{VO}_{2}$ [40]. A temperature dependence of XANES spectra was also observed at the K-edge of oxygen in water [41] and in doped $\mathrm{LaMnO}_{3}$ [42, 43]. Finally, the MahanNozières-Dominics singularity can also give rise to a temperature dependence of the x-ray absorption spectra of metals (see Ref. [44] for a review).

We come now to our experimental temperature dependence at the K-edge of titanium in rutile.

\section{Experiment}

The x-ray absorption experiments were performed at the D11 (energy dispersive) and at the EXAFSII stations of the DCI storage ring of the Laboratoire pour l'Utilisation du Rayonnement Synchrotron in Orsay (France).

A rutile single-crystal plate $(9 \mathrm{~mm} \times 4 \mathrm{~mm} \times 50 \mu \mathrm{m})$ was measured at the D11 station in the transmission mode. The crystal plate was placed inside a liquid-helium cryostat operating between $4.2 \mathrm{~K}$ and $300 \mathrm{~K}$. Measurements were carried out for two orientations, with the (110) face of the crystal perpendicular to the x-ray beam and the $c$-axis either parallel or perpendicular to the linear polarization vector of the beam. The polychromator consisted in a curved $\mathrm{Si}(111)$ crystal focusing the X-ray beam at the center of the cryostat sample holder. Higher harmonics were rejected by a $\mathrm{SiO}_{2}$ plane mirror. The x-ray intensity was measured by a photodiode array detector. Each spectrum was obtained as a result of four measurements: $I_{0}$ (without sample and with the beam), $I_{0 \text { black }}$ (without sample and without beam), $I$ (with sample and with the

beam), $I_{\text {black }}$ (with sample and without beam). The absorption spectrum was then obtained from the formula $\sigma=\log \left(I_{0}-I_{0 \text { black }}\right)-\log \left(I-I_{\text {black }}\right)$. The x-ray energy corresponding to each detector pixel was determined by comparing the spectra with a spectrum measured on a two-crystal monochromator beamline. The energy resolution was typically $0.8 \mathrm{eV}$.

Our preliminary study [15] showed us that the pre-edge structure could exhibit a low signal to noise $(\mathrm{S} / \mathrm{N})$ ratio when the crystal thickness was optimized for the edgejump. Therefore, we optimized the crystal thickness for the pre-edge structure and cut an approximately $50 \mu \mathrm{m}$ thick crystal plate. As a consequence, we obtained excellent spectra in the pre-edge region but the XANES spectra after the edge had a rather low $\mathrm{S} / \mathrm{N}$ ratio and, for each polarization direction, we normalized the spectrum at the inflection point of the absorption edge instead of at the edge jump.

To check the validity of this procedure, we carried out additional experiments at the EXAFSII station. The experimental equipment consisted of a two-crystal 

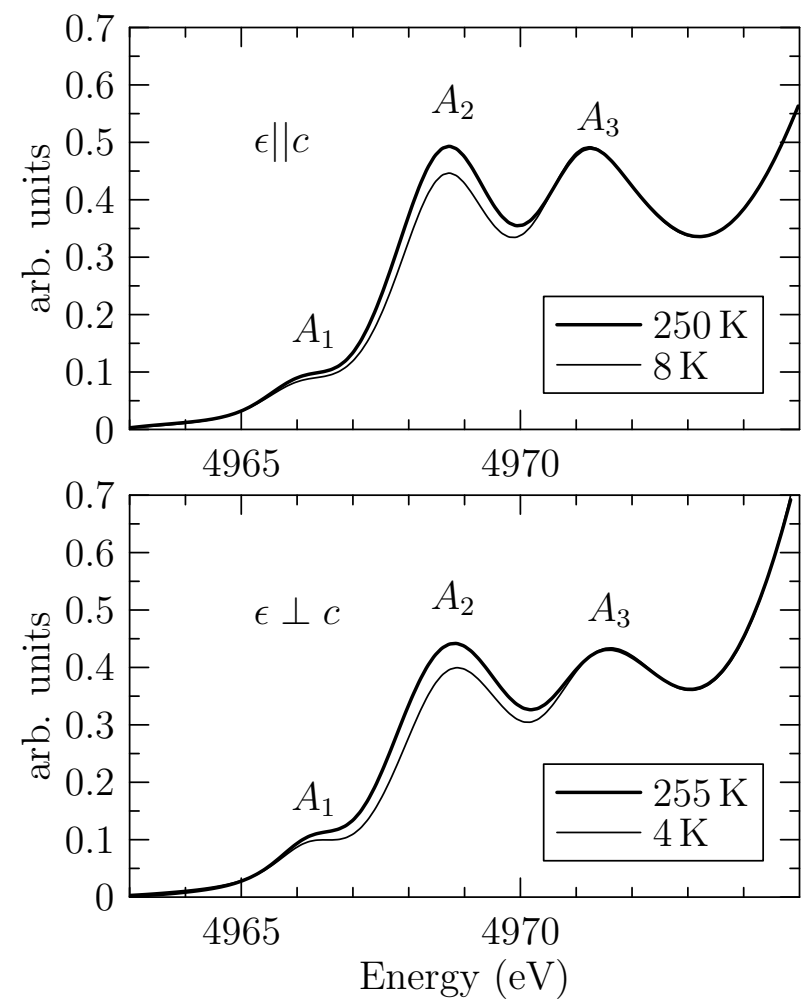

Figure 1. Temperature variation of the pre-edge peaks at the K-edge of titanium in rutile, with the x-ray polarization vector parallel and perpendicular to the $c$-axis of the crystal.

$\mathrm{Si}(311)$ monochromator, an ionisation chamber to measure the incident beam and an electron-yield detector. We measured a bulk rutile single crystal with the (110) face perpendicular to the x-ray beam and with the $c$-axis of the crystal either parallel or perpendicular to the $\mathrm{x}$-ray polarization vector. The $\mathrm{S} / \mathrm{N}$ ratio of the pre-edge region was comparable to that of the Ti K-edge spectra of rutile measured on the same beamline in similar conditions [45, 46]. The experimental spectra were normalized by the standard procedure and, as in our previous work [15], the temperature dependence was found to be negligible except in the pre-edge region. Moreover, the observed spectra and temperature dependence agreed well with the transmission experiments of the D11 station. In the present paper we show only the results of the transmission experiments because of their better $\mathrm{S} / \mathrm{N}$ ratio.

\section{Experimental results}

Figure 1 shows the pre-edge features of rutile recorded at different temperatures with the polarization vector perpendicular and parallel to the crystal $c$-axis. A decrease of the first two peaks $A_{1}$ and $A_{2}$ is observed at low temperature, whereas the third peak $\mathrm{A}_{3}$ does not show any significant variation. It is important to notice that temperature induces a change in the intensity but not in the energy position of the peaks. 


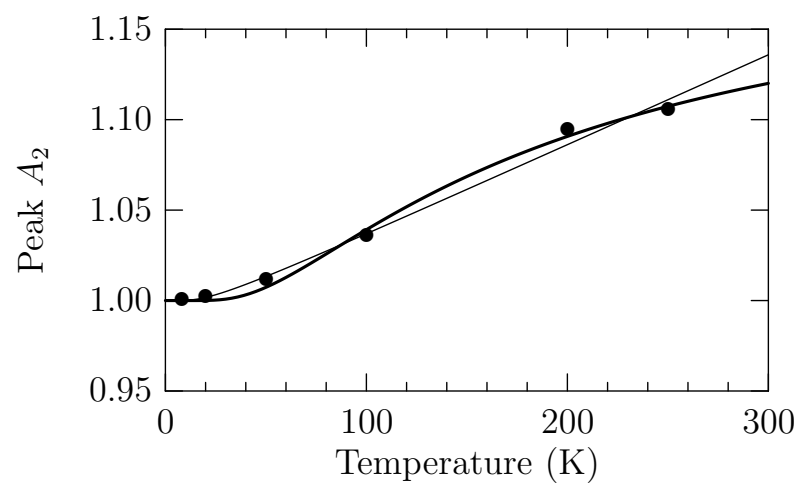

Figure 2. Relative intensity of peak $A_{2}$ as a function of temperature for $\epsilon \| c$, normalized to 1 at $8 \mathrm{~K}$. Dots: experimental results; thick solid line: fit to the function $a\left(1+\mathrm{e}^{-\theta / T}\right)+(1-a)$; thin solid line: fit to the function $a \cosh (\theta / 2 T)+(1-a)$.

The physical origin of the pre-edge peaks of titanium in rutile is well known [47]. Peaks $A_{1}$ and $A_{2}$ correspond to electric quadrupole transitions towards $3 d$ states of titanium with $t_{2 g}$ and $e_{g}$ symmetry, respectively. Therefore, the peaks that vary with temperature are also the peaks corresponding to quadrupole transitions.

In the optical range, the effect of temperature is usually described by a simple model developed by Holmes and McClure [48, 49, 50, in which the intensity of the vibronic peak varies as $1+e^{-\theta / T}$, where $\theta$ is the energy of the first vibrational level. Figure 2 shows the variation of the $A_{2}$ peak with temperature, fitted to the function $a\left(1+e^{-\theta / T}\right)+(1-a)$, where $a\left(1+e^{-\theta / T}\right)$ represents the fraction of the $A_{2}$ peak that is purely vibrational and $1-a$ the fraction that is due to electric quadrupole transitions (and to the possible tail of the electric dipole peak $A_{3}$ ).

The result of the fit is $a=0.21$ and $\theta=168 K \pm 10 K$. Note that the value of $\theta$ compares favorably to the energy of the first odd vibrational level at the $\Gamma$ point obtained by ab initio calculations (168 K [51], $150 \mathrm{~K}$ [26], $169 \mathrm{~K}$ [25] or $181 \mathrm{~K}$ [23]) or by neutron scattering $163 \mathrm{~K}$ [52]. However, the simplicity of the Holmes and McClure model implies that the quality of this agreement is probably fortuitous. Indeed, an alternative singlemode model is sometimes used [53, 54, 55, 56], for which the temperature dependence is $\operatorname{coth} \theta / 2 T$. For this second model the fit gives $\theta=58 K \pm 5 K$ and $a=0.014$.

\section{Interpretation}

It remains to understand why only the first two peaks vary with temperature while the rest of the XANES spectrum remains constant. We first describe the arguments that are usually given to explain the absence of temperature dependence of XANES spectra. Then, we show why, in some circumstances, this independence can be broken. 


\subsection{The temperature independence of XANES spectra}

There are many reasons to believe that the pre-edge features of x-ray absorption spectra do not depend on temperature in the absence of structural transition. The first reason comes from the temperature dependence of the EXAFS part of x-ray absorption spectra, which is represented by a Debye-Waller factor $\mathrm{e}^{-2 k^{2} \sigma}$ in the EXAFS formula. The DebyeWaller factor accurately describes the temperature dependence of XAS in crystals, although it has to be supplemented with higher order cumulants in disordered materials. Moreover, it is well understood because it can be calculated $a b$ initio with a good agreement with experiment [57, 58, 59, 60, 61]. If we use this factor to describes the temperature dependence near the edge, we must take an energy very close to the Fermi energy, so that $k$ is very small and the factor is close to unity.

Of course, near the edge, the effect of temperature is not supposed to be described by a Debye-Waller factor and we must use a more sophisticated approach. Natoli's rule [62] gives good results near the edge. However, it describes an energy shift through the equation $k R=$ Constant, and we do not observe any energy shift. More elaborate theoretical analyses were carried out. Brouder and Goulon [63, 64] used Lie group theory to describe the influence of a displacement on the multiple-scattering operator. However, the temperature evoluation is expected to be small near the edge, essentially because of Natoli's rule. Poiarkova and Rehr [57] extended the Debye-Waller factor to multiplescattering paths. Their formalism is not really valid in the pre-edge region, but if we try to extend it we find a very small temperature dependence because of the presence of the $k^{2}$ factor in the exponent. Fujikawa [65, 66] used Schwinger's technique to calculate the effect of the Franck-Condon factors on XAFS. He concluded that this effect was not important. In a later work [67], he investigated the effect of temperature through the Keldysh approach to nonequilibrium systems. He found that thermal vibrations could be represented by a convolution with the phonon spectral function. His result is valid in the pre-edge region but leads to a small temperature effect. Moreover, the convolution should give rise to a broadening of the pre-edge peaks with temperature. Again, this is not compatible with our experimental results. A further elaboration of his approach 68] lead to similar results.

We can try to take vibrations into account by coming back to the BornOppenheimer approximation and writing the initial and final wavefunctions as a product of a vibrational and an electronic function. However, this approach looks like a dead end if we consider the work by Mäder and Baroni [69] who showed that, at the K-edge of carbon, the vibrations in the final state are strongly anharmonic and are deeply affected by the presence of the core-hole. Therefore, we are not allowed to consider the vibrations as similar in the initial and final states and we cannot use the harmonic approximation.

Ankudinov and Rehr [70] brought some hope by showing that the S K-edge spectrum of $\mathrm{SF}_{6}$ is closer to experiment when the atomic positions are slightly shifted

with respect to the equilibrium position. But, as can be seen in their figure, atomic displacements shift the position of the main lines and this shift is not experimentally 
observed.

\subsection{The temperature dependence of XANES spectra}

We recently proposed a model that enables us to understand the observed temperature dependence [71]. Although a detailed account of this model would be beyond the scope of the present paper, we can give a physical description of the underlying physics.

We start from the Born-Oppenheimer approximation where the wavefunctions of the electron+nuclei system is the product of a vibrational function by a solution of the Schrödinger equation for clamped nuclei. The energy of these wavefunctions do not depend on the position of the nuclei (as the eigenvalues of the Schrödinger equation for an electron in a potential do not depend on position). The transitions are made between these wavefunctions. If we assume that the vibrational energies are small with respect to experimental resolution, we can sum over the final state vibrational functions and we obtain an average over the vibrational function of the initial state of transitions for which the transition energy do not depend on the atomic positions. This explains why the peak positions do not move while they move if we calculate the spectrum of a distorted structure.

The second step of the model consists in making a different approximation for the initial and the final states. The initial state is taken to be the core state centered at the position specified by the vibrational wave function. For the final state, we make the crude Born-Oppenheimer approximation, where the electronic wavefunction is assumed independent of the position of the absorbing atom. Then, the cross-section boils down to an average of the x-ray absorption spectra for a shifted core wavefunction (with fixed energies). What happens next can be sketched by an oversimplified model of the shifted core wavefunction. We assume that the displacement $\mathbf{R}$ is small compared to the electronic variable $r$ and we obtain, to first order in $\mathbf{R}$ and for a spherical core state $\phi_{0}(r)$, the shifted function

$$
\phi_{0}(|\mathbf{r}-\mathbf{R}|) \simeq \phi_{0}(r)-\frac{\mathbf{r} \cdot \mathbf{R}}{r} \phi_{0}^{\prime}(r)
$$

When multiplied by $\epsilon \cdot \mathbf{r}$, the additional term gives us a factor $\epsilon \cdot \mathbf{r R} \cdot \mathbf{r}$ that can be transformed into the sum of a monopole term proportional to $(\epsilon \cdot \mathbf{R}) r^{2}$ and a quadrupole term. The monopole term gives rise to transitions towards $s$ states, the quadrupole term to transition towards $d$ states. The transition towards $s$ states are observed at the aluminium or silicon K-edge [71], the transitions towards $d$ states are observed at the K-edge of transition metals because of the presence of a strong density of $d$ states. This explains why the temperature variation occurs at the position of the quadrupole peaks. Finally, the fact the temperature-dependent pre-edge peaks grow with temperature is due to the corresponding increase in thermal vibration amplitudes.

Of course, eq. (1) is not sufficient because the integration over $\mathbf{r}$ includes also a region where $r<R$. The full theory [71] is more complex but the physical idea is the same. 


\section{Conclusion}

In this paper, we have presented the temperature-dependence of pre-edge features at the K-edge of titanium in rutile. This temperature dependence is not due to a phase transition or to a static distortion of the titanium site.

The temperature dependence changes only the intensities of the peaks and not their positions. Moreover, the peaks that vary with temperature are the electric quadrupole peaks of the spectrum. An explanation of this behavior was given in terms of the dynamic displacement of the absorbing atom.

Two conditions turn out to be crucial to observe temperature-dependent pre-edge peaks at the K-edge : (i) a large density of $d$ states below the $p$ states, so that the transitions to final $d$ states are significant and visible; (ii) the existence of lowenergy vibrational modes, so that the temperature effect can be observed at reasonable temperatures. Both of these conditions are satisfied in rutile and in perovskites containing transition metals. In that case, the temperature dependence provides information on the local vibrations around the absorbing atom. This can be particularly useful to investigate the vibrations of transition metal impurities.

\section{Acknowledgments}

We thank Delphine Cabaret for very constructive comments.

\section{References}

[1] Yamamoto T 2008 Assignment of pre-edge peaks in K-edge x-ray absorption spectra of 3d transition metal compounds: electric dipole or quadrupole? X-ray spectrometry $37572-84$

[2] Chalmin E, Farges F and Jr G E B 2009 A pre-edge analysis of Mn K-edge XANES spectra to help determine the speciation of manganese in minerals and glasses Contrib. Mineral. Petrol. $157111-26$

[3] Arfaoui M, Cabaret D, Longa S D, Seitsonen A and Mauri F 2007 First-principles full-potential calculations of the Fe $\mathrm{K}$ pre-edge and near-edge structure in carbonmonoxy-myoglobin in B Hedman and P Painetta, eds., AIP Conference Proceedings vol. 882 (AIP) pp. 331-3

[4] Getty K, Delgado-Jaime M U and Kennepohl P 2008 Assignment of pre-edge features in the Ru K-edge x-ray absorption spectra of organometallic ruthenium complexes Inorg. Chim. Acta $\mathbf{3 6 1}$ $1059-65$

[5] Gougoussis C, Calandra M, Seitsonen A, Brouder C, Shukla A and Mauri F 2009 Intrinsic charge transfer gap in $\mathrm{NiO}$ from Ni K-edge x-ray absorption spectroscopy Phys. Rev. B 79045118

[6] Vedrinskii R V, Kraizman V L, Lemeshko M P, Nazarenko E S, Novakovich A A, Reznichenko L A, Fokin V N and Shuvaeva V A 2009 Local atomic structure of niobates and titanates from x-ray absorption spectroscopic data Phys. Solid State 51 1394-8

[7] Arrio M A, Rossano S, Brouder C, Galoisy L and Calas G 2000 Calculation of multipole transitions at the Fe K pre-edge through p-d hybridization in the ligand field multiplet model Europhys. Lett. $51454-60$

[8] de Groot F M F, Vankó G and Glatzel P 2009 The 1s x-ray absorption pre-edge structures in transition metal oxides J. Phys.: Condens. Matter 21104207

[9] Shirley E 2004 Ti 1s pre-edge features in rutile: a Bethe-Salpeter calculation J. Elect. Spect. Relat. Phenom. 136 77-83 
[10] Wu Z Y, Natoli C R, Marcelli A, Paris E, Bianconi A and Saini N L 2003 Unified interpretation of pre-edge x-ray absorption fine structures in $3 \mathrm{~d}$ transition metal compounds in A Bianconi, A Marcelli and N L Saini, eds., AIP Conference Proceedings vol. 652 (AIP) pp. 497-506

[11] Juhin A, Brouder C, Arrio M A, Cabaret D, Sainctavit P, Balan E, Bordage A, Calas G, Eeckhout $\mathrm{S}$ G and Glatzel P $2008 \mathrm{X}$-ray linear dichroism in cubic compounds: the case of $\mathrm{Cr}^{3+}$ in $\mathrm{MgAl}_{2} \mathrm{O}_{4}$ Phys. Rev. B $\mathbf{7 8} 195103$

[12] Vedrinskii R V, Kraizman V L, Novakovich A A, Demekhin P V, Urazhdin S V, Ravel B and Stern E A 1997 Pre-edge fine structure (PEFS) of the K-XAS for the 3d atoms in coumpounds: A new tool for quantitative atomic structure determination J. Physique 7C2 107-10

[13] Vedrinskii R V, Kraizman V L, Novakovich A A, Demekhin P V and Urazhdin S V 1998 Preedge fine structure of the $3 d$ atom $\mathrm{K}$ x-ray absorption spectra and quantitative atomic structure determinations for ferroelectric perovskite structure crystals J. Phys.: Condens. Matter 10 9561-9580

[14] Vedrinskii R V, Nazarenko E S, Lemeshko M P, Nassif V, Proux O, Novakovich A A and Joly Y 2006 Temperature dependent XAFS studies of local atomic structure of the perovskite-type zirconates Phys. Rev. B $\mathbf{7 3} 134109$

[15] Durmeyer O, Kappler J P, Beaurepaire E, Heintz J M and Drillon M 1990 Ti K XANES in superconducting $\mathrm{LiTi}_{2} \mathrm{O}_{4}$ and related compounds J. Phys.: Condens. Matt. 2 6127-36

[16] Burdett J K, Hughbanks T, Miller G J, Jr J W R and Smith J V 1987 Structural-electronic relationships in inorganic solids: powder neutron diffraction studies of the rutile and anatase polymorphs of titanium dioxide at 15 and 295 K J. Am. Chem. Soc. 109 3639-46

[17] Howard C J, Sabine T M and Dickson F 1991 Structure and thermal parameters for rutile and anatase Acta Cryst. B $\mathbf{4 7}$ 462-8

[18] Sakata M, Uno T, Takata M and Howard C J 1993 Maximum-entropy-method analysis of neutrondiffraction data J. Appl. Cryst. 26 159-65

[19] Sabine T M, Kennedy B J, Garrett R F, Goran G J and Cookson D J 1995 The performance of the Australian powder diffractometer at the photon-factory, Japan J. Appl. Cryst. 28 513-7

[20] Kumazawa S, Takata M and Sakata M 1995 An accurate determination of the thermal vibration of rutile from the nuclear density distribution of the maximum-entropy analysis Acta Cryst. A $51651-8$

[21] Jiang B, Zuo J M, Jiang N, O'Keeffe M and Spence J C H 2003 Charge density and chemical bonding in rutile, $\mathrm{TiO}_{2}$ Acta Cryst. A $\mathbf{5 9} 341-50$

[22] Montanari B and Harrison N M 2004 Pressure-induced instabilities in bulk $\mathrm{TiO}_{2}$ rutile $J$. Phys.: Condens. Matter 16 273-92

[23] Rignanese G M, Rocquefelte X, Gonze X and Pasquarello A 2005 Titanium oxides and silicates as high- $\kappa$ dielectrics: A first-principles investigation Int. J. Quant. Chem. 101 793-801

[24] Rignanese G M, Rocquefelte X, Gonze X and Pasquarello A 2005 Erratum: Titanium oxides and silicates as high- $\kappa$ dielectrics: A first-principles investigation Int. J. Quant. Chem. 103354

[25] Sikora R $2005 \mathrm{Ab}$ initio study of phonons in the rutile structure of $\mathrm{TiO}_{2}$ J. Phys. Chem. Solids 66 1069-73

[26] Montanari B and Harrison N M 2002 Lattice dynamics of $\mathrm{TiO}_{2}$ rutile: influence of gradient corrections in density functional calculations Chem. Phys. Lett. 364 528-34

[27] Ravel B, Stern E A, Yacobi Y and Dogan F 1993 Lead titanate is not a classic case of a displacive ferroelectric phase transition Jpn. J. Appl. Phys. Suppl. 32-2 782-784

[28] Engel E, Dreizler R and Malzacher P 1987 Density functionals on the basis of the relativistic field theory J. Physique 48C2 321-8

[29] Sato K, Miyanaga T, Ikeda S and Diop D 2005 XAFS study of local structure change in perovskite titanates Physica Scripta T115 359-61

[30] Hashimoto T, Yoshiasa A, Okube M, Okudera H and Nakatsuka A 2007 Temperature dependence of XANES spectra for $\mathrm{ATiO}_{3}, \mathrm{~A}_{2} \mathrm{TiO}_{4}$ and $\mathrm{TiO}_{2}$ compounds with structural phase transitions in B Hedman and P Painetta, eds., AIP Conference Proceedings vol. 882 (AIP) pp. 428-30 
[31] Nozawa S, Iwazumi T and Osawa H 2005 Direct observation of the quantum fluctuation controlled by ultraviolet irradiation in $\mathrm{SrTiO}_{3}$ Phys. Rev. B $\mathbf{7 2} 121101$

[32] Shuvaeva V A, Yanagi K, Yagi K, Sakuae K and Terauchi H 1999 Polarized XAFS study of the atomic displacements and phase transitions in $\mathrm{KNbO}_{3}$ J. Synchrotron Rad. 6 367-9

[33] Shuvaeva V A, Pirog I, Azuma Y, Yagi K, Sakuae K, Terauchi H, Raevskii I P, Zhuchkov K and Antipin M Y 2003 The local structure of mixed-ion perovskites J. Phys.: Condens. Matt. 15 2413-21

[34] Shuvaeva V A, Azuma Y, Yagi K, Sakuae K and Terauchi H 2001 Polarized XAFS study of high-temperature phases of $\mathrm{NaNbO}_{3}$ J. Synchrotron Rad. 8 833-35

[35] Shuvaeva V A, Azuma Y, andK Yagi I P R, Sakuae K and Terauchi H 2004 The local structure of $\mathrm{PbIn}_{1 / 2} \mathrm{Nb}_{1 / 2} \mathrm{O}_{3}$ Ferroelectrics 299 103-8

[36] Qian Q, Tyson T A, Kao C C, Croft M, Cheong S W and Greenblatt M 2000 Thermal effects in the x-ray spectra of $\mathrm{La}_{1-x} \mathrm{Ca}_{x} \mathrm{MnO}_{3}$ Phys. Rev. B 62 13472-13481

[37] Bridges F, Booth C H, Kwei G H, Neumeier J J and Sawatzky G A 2000 Temperature dependent changes of the Mn $3 d$ and $4 p$ bands near Tc in colossal magnetoresistance systems: XANES study of $\mathrm{La}_{1-x} \mathrm{Ca}_{x} \mathrm{MnO}_{3}$ Phys. Rev. B 61 R9237-R9240

[38] Deb A, Ralph J M, Cairns E J and Bergmann U 2006 Characterization of $\mathrm{La}_{0.8} \mathrm{Sr}_{0.2} \mathrm{FeO}_{3-\delta}$ ] and $\left.\mathrm{La}_{0.7} \mathrm{Sr}_{0.2} \mathrm{FeO}_{3-\delta}\right]$ as a function of temperature by x-ray absorption spectroscopy Phys. Rev. $B$ 73115114

[39] Hidaka M, Tokiwa N, Oda M, Choi J Y and Lee J M 2003 Electronic states near Fermi level in superconductors $\mathrm{La}_{2-} \mathrm{Sr}_{x} \mathrm{CuO}_{4}$ by means of x-ray absorption spectra near $\mathrm{Cu}-\mathrm{K}$ and La-L edges Phase Transitions 76 905-21

[40] Poumellec B, Cortes R, Loisy E and Berthon J 1994 Polarization dependence of the V K edge in $\mathrm{VO}_{2}$ through the phase transition at $68{ }^{\circ} \mathrm{C}$ Phys. Status. Solidi $b \mathbf{1 8 3} 335-50$

[41] Wernet P, Nordlund D, Bergmann U, Cavarelli M, Odelius M, Ogasawara H, Näslund L A, Hirsch T K, Ojamäe L, Glatzel P, Pettersson L G M and Nilsson A 2004 The structure of the first coordination shell in liquid water Science 304 995-9

[42] Mannella N, Rosenhahn A, Watanabe M, Sell B, Nambu A, Ritchey S, Arenholz E, Young A, Tomioka Y and Fadley C S 2005 Temperature-dependent x-ray absorption spectroscopy of colossal magnetoresistive perovskites Phys. Rev. B $\mathbf{7 1} 125117$

[43] Tsai Y T, Chang W J, Huang S W, Lin J Y, Lee J Y, Chen J M, Wu K H, Uen T M, Gou Y S and Juang J Y 2009 Temperature-dependent x-ray absorption near edge spectroscopy of doped $\mathrm{LaMnO}_{3}$ : Ion-size and the Jahn-Teller distortion effects Physica B: Condensed Matter 4041404 $-1408$

[44] Ohtaka K and Tanabe Y 1990 The soft-x-ray edge problem Rev. Mod. Phys. 62 929-91

[45] Brouder C, Kappler J P and Beaurepaire E 1990 Theory and application of angle-resolved x-ray absorption spectra in A Balerna, E Bernieri and S Mobilio, eds., 2nd European Conference on Progress in X-Ray Synchrotron Radiation Research vol. 25 of Conference Proceedings (Bologna: Italian Physical Society) pp. 19-22

[46] Poumellec B, Cortes R, Tourillon G and Berthon J 1991 Angular dependence of the Ti K edge in rutile $\mathrm{TiO}_{2}$ Phys. Status. Solidi b 164 319-26

[47] Joly Y, Cabaret D, Renevier H and Natoli C R 1999 Electron population analysis by full-potential x-ray absorption simulations Phys. Rev. Lett. 82 2398-401

[48] Holmes O G and McClure D S 1957 Optical spectra of hydrated ions of the transition metals $J$. Chem. Phys. 26 1686-1694

[49] McClure D S 1959 Electronic spectra of molecules and ions in crystals. Part II. Spectra of ions in crystals in H Ehrenreich and D Turnbull, eds., Solid State Physics vol. 9 (New York: Academic Press) pp. 399-525

[50] Triest M, Davis M J and Reber C 1999 Absorption spectroscopy and time-dependent theoretical calculation of the intervalence band of $\left[\mathrm{Fe}^{\mathrm{II}} \mathrm{Fe}{ }^{\mathrm{III}} \mathrm{BPMP}(\mathrm{OPr})_{2}\left(\mathrm{BPh}_{4}\right)_{2}\right.$, a localized mixed-valence compound with a nonlinear metal-bridging ligand-metal core New J. Chem. 23 425-31 
[51] Lee C, Ghosez P and Gonze X 1994 Lattice dynamics and dielectric properties of incipient ferroelectric $\mathrm{TiO}_{2}$ rutile Phys. Rev. B 50 13379-87

[52] Traylor J G, Smith H G, Nicklow R M and Wilkinson M K 1971 Lattice dynamics of rutile Phys. Rev. B 3 3457-72

[53] Pisarev R V and Prokhorova S D 1969 The temperature dependence of optical absorption in the antiferromagnet $\mathrm{KNiF}_{3}$ Sov. Phys. Solid State 10 1668-72

[54] Cieślak-Golonka M, Bartecki A and Sinha S P 1980 Vibrational fine structure in the electronic spectra of transition metal compounds. An experimental study Coord. Chem. Rev. 31 251-88

[55] McDonald R G and Hitchman M A 1986 Electronic "d-d" spectra of the planar $\mathrm{CuCl}_{4}^{2-}$ ions in bis(methadonium) tetrachlorocuprate(ii) and bis(creatinium) tetrachlorocuprate(ii): Analysis of the temperature dependence and vibrational fine structure Inorg. Chem. 25 3273-81

[56] Taran M N, Langer K, Platonov A N and Indutny V V 1994 Optical absorption investigation of $\mathrm{Cr}^{3+}$ ion-bearing minerals in the temperature range 77-797k Phys. Chem. Minerals 21 360-72

[57] Poiarkova A V and Rehr J J 1999 Multiple-scattering x-ray-absorption fine-structure Debye-Waller factor calculations Phys. Rev. B 59 948-57

[58] Chun W J, Ijima K, Ohminami Y, Suzuki S and Asakura K 2004 Theoretical Debye-Waller factors of $\alpha-\mathrm{MoO}_{3}$ estimated by an equation-of-motion method J. Synchrotron Rad. 11 291-4

[59] Vaccari M and Fornasini P 2005 Thermal effects on EXAFS: Ensemble averages and real-space approach Phys. Rev. B $\mathbf{7 2} 092301$

[60] Dimakis N 2007 Ab initio calculation of XAFS Debye-Waller factors for crystalline materials in B Hedman and P Painetta, eds., AIP Conference Proceedings vol. 882 (AIP) pp. 126-8

[61] Vila F D, Rehr J J, Rossner H H and Krappe H J 2007 Theoretical x-ray absorption Debye-Waller factors Phys. Rev. B $\mathbf{7 6} 014301$

[62] Natoli C R 1983 Near edge absorption structure in the framework of the multiple scattering model. Potential resonance or barrier effects? in EXAFS and Near Edge Structure vol. 27 of Springer Series in Chemical Physics (Berlin: Springer-Verlag) pp. 43-56

[63] Brouder C and Goulon J 1989 Influence of atomic displacements on XAFS Physica B 158 351-4

[64] Brouder C 1992 Lie group calculation of the Green function of disordered systems in W H Butler, P H Dederichs, A Gonis and R L Weaver, eds., Applications of Multiple Scattering Theory to Materials Science vol. 253 of Materials Research Society Symposium Proceedings (Pittsburgh: Materials Research Society) pp. 411-6

[65] Fujikawa T 1996 Debye-Waller and Condon factors in XAFS J. Elect. Spect. Relat. Phenom. 79 $25-28$

[66] Fujikawa T 1996 Theory of atomic displacement effects induced by core-hole production on EXAFS J. Phys. Soc. Japan 65 87-94

[67] Fujikawa T 1999 XAFS theory at zero and finite temperature J. Phys. Soc. Japan 68 2444-56

[68] Arai H, Ueno N and Fujikawa T 2007 Theory of electron-phonon interaction in XAFS and other spectroscopies in B Hedman and P Painetta, eds., AIP Conference Proceedings vol. 882 (AIP) pp. $108-10$

[69] Mäder K A and Baroni S 1997 Vibrational broadening of x-ray emission spectra: A first-principles study on diamond Phys. Rev. B 55 9649-58

[70] Ankudinov A L and Rehr J J 2005 Nonspherical potential, vibronic and local field effects in x-ray absorption Physica Scripta T115 24-7

[71] Brouder C, Cabaret D, Juhin A and Sainctavit P, Effect of vibrations on the pre-edge features of x-ray absorption spectra to be published arXiv:0912.0792 